\title{
Novo financiamento para uma nova Atenção Primária à Saúde no Brasil
}

\author{
New funding for a new Brazilian Primary Health Care
}

Erno Harzheim (https://orcid.org/0000-0002-8919-7916) ${ }^{1}$

Otávio Pereira D’Avila (https://orcid.org/0000-0003-1852-7858) ${ }^{1}$

Daniela de Carvalho Ribeiro (https://orcid.org/0000-0002-3055-0041) ${ }^{1}$

Larissa Gabrielle Ramos (https://orcid.org/0000-0002-3441-0951) ${ }^{1}$

Lariça Emiliano da Silva (https://orcid.org/0000-0001-7501-1095) ${ }^{1}$

Caroline Martins José dos Santos (http://orcid.org/0000-0003-3384-2637) ${ }^{1}$

Luis Gustavo Mello Costa (https://orcid.org/0000-0002-9678-0173) ${ }^{1}$

Carlo Roberto Hackmann da Cunha (https://orcid.org/0000-0002-3545-5801) ${ }^{1}$

Lucas Alexandre Pedebos (https://orcid.org/0000-0001-6663-352X) ${ }^{1}$
${ }^{1}$ Secretaria de Atenção

Primária à Saúde, Ministério

da Saúde. Esplanada dos

Ministérios, Bloco G,

Térreo. 70058-900 Brasília

DF Brasil.

eharzheim@hcpa.edu.br.

\begin{abstract}
This paper aims to present a debate on the new Brazilian Primary Health Care (PHC) funding policy. We consulted the national and international literature, and we involved municipal, state, and federal PHC managers to develop the payment method. The proposed final model is based on weighted capitation, payment-for-performance, and incentive for strategic actions. Capitation is weighted by the socioeconomic vulnerability, demographic aspects, and municipal adjustment, the payment-for-performance consists of an entire set of 21 indicators, and incentives for strategic actions were facilitated from the maintenance of some specific programs. The results of the simulations pointed to low registration (90 million Brazilians) for the currently estimated coverage (148,674,300 Brazilians). Moreover, they showed an immediate increase in financial resources for 4,200 Brazilian municipalities. We observed that the funding proposal brings Brazilian PHC into the 21st century, points to the strengthening of PHC attributes, and materializes the principles of universality and equity of the Unified Health System.
\end{abstract}

Key words Health System Financing, Primary Health Care, Capitation Fee, Efficiency, Health Equity
Resumo O objetivo desse artigo é apresentar um debate sobre a nova politica de financiamento para Atenção Primária à Saúde (APS) no Brasil. Para desenvolvimento do método de pagamento foi realizado consulta da literatura nacional e internacional, além do envolvimento de gestores municipais, estaduais e federais da APS. O modelo final proposto é baseado em Capitação ponderada; Pagamento por desempenho; Incentivo para Ações Estratégicas. A capitação é ponderada por vulnerabilidade socioeconômica, aspectos demográficos e ajuste municipal, o pagamento por desempenho composto por um conjunto total de 21 indicadores e incentivos a ações estratégicas foi possivel a partir da manutenção de alguns programas especificos. Os resultados das simulações apontaram para um baixo cadastro (90 milhões de brasileiros) para a cobertura estimada atual (148.674.300 milhões de brasileiros). Além disso, demonstraram um incremento imediato de recursos financeiros para 4.200 municípios brasileiros. Observa-se que a proposta do financiamento traz a APS brasileira para o século XXI, aponta para o fortalecimento dos atributos da APS e torna concreto os princípios de universalidade e equidade do Sistema Único de Saúde.

Palavras-chave Financiamento do Sistema de Saúde, Atenção Primária à Saúde, Taxa de Capitação, Eficiência, Equidade em Saúde 


\section{Introdução}

A regulamentação do Sistema Único de Saúde (SUS) foi estabelecida pela Lei 8.080 de 19 de setembro de $1990^{1}$. Para além dos seus princípios e diretrizes a referida lei também apresentava as competências de cada ente federativo com relação a gestão do SUS. Especificamente, quanto ao financiamento do SUS o Art. 35 estabelecia que para os valores a serem transferidos a Estados, Distrito Federal e Municípios, seria utilizada a combinação dos seguintes critérios: perfil demográfico da região; perfil epidemiológico da população a ser coberta; características quantitativas e qualitativas da rede de saúde na área; desempenho técnico, econômico e financeiro no período anterior; níveis de participação do setor saúde nos orçamentos estaduais e municipais; previsão do plano quinquenal de investimentos da rede; ressarcimento do atendimento a serviços prestados para outras esferas de governo. Mais tarde, com promulgação da Lei de 8.142 de 28 de dezembro de $1990^{2}$, houve a definição dos mecanismos de transferências intergovernamentais de recursos financeiros na área da saúde, estabelecendo marco legislativo para o financiamento do SUS. Em 1993, a Norma Operacional Básica (NOB) $01 / 1993^{3}$ propôs pequenos avanços na autonomia e flexibilização para as gestões municipais.

Nesse ínterim marcado por avanços na consolidação do SUS, a NOB 01/964, apontava para uma reorganização do modelo de atenção à Saúde apresentando papéis de cada ente federado, instrumentos gerenciais, mecanismos e fluxos de financiamento, reduzindo progressivamente a remuneração por produção de serviços e ampliando as transferências de caráter global, fundo a fundo. Nesta NOB, houve a instituição do Piso de Atenção Básica (PAB), que definia a transferência de recursos financeiros regulares para a Atenção Básica a partir de um valor per capita, seguindo critérios estabelecidos na Lei $8.080^{1}$. Além disso, a NOB apresentava uma variação para a transferência de recursos via $\mathrm{PAB}$. Aqueles municípios que aderiam ao Programa de Saúde da Família (PSF) ou ao Programa de Agentes Comunitários de Saúde (PACS), recebiam um volume adicional de recursos integrados ao PAB. Com o estabelecimento do PAB fixo e variável, sendo o fixo com base em valor per capita e o variável relativo a adesão a programas específicos ${ }^{5}$, estabeleceu-se um conjunto de serviços e atribuições claras para a Atenção Primária à Saúde (APS) no Brasil. Em 1998, o Manual para Organização da Atenção
Básica ${ }^{6}$ estabeleceu diretrizes, responsabilidades, indicadores de monitoramento e critérios para uso desses recursos.

O início dos anos 2000 continuaram marcados por transformações no financiamento do SUS e, principalmente, da APS, mas de menor monta. A Norma Operacional da Assistência à Saúde (NOAS-SUS) 01/2000 ${ }^{7}$, instituiu a gestão plena da Atenção Básica/APS ampliada aumentando as responsabilidades assistenciais da APS no país e instituindo o PAB Ampliado para os municípios que aderissem. Com o passar dos anos o PAB Ampliado acabou sendo incorporado ao PAB fixo.

Nesse período, foi reforçada a fragmentação dos modelos de repasses a partir do estabelecimento de blocos de financiamento ${ }^{8}$. Essa fragmentação sequestrou a autonomia dos gestores municipais que ficaram presos aos repasses inflexíveis e pouco eficientes para as realidades municipais. A Portaria 3.992, de 28 de dezembro de $2017^{9}$, alterou esse cenário instituindo apenas dois blocos: custeio e investimento. Dessa forma, gestores municipais retomaram parte de sua autonomia ao recuperar essa desvinculação financeira e, com isso, a possibilidade de qualificar a gestão com base na percepção das necessidades. Essas alterações foram ao encontro da Lei Complementar $\mathrm{n}^{\circ} 141$, de 13 janeiro de $2012^{10}$, que busca transparência para os critérios de rateio entre os entes federados ao incluir critérios socioeconômicos, epidemiológicos, demográficos, espaciais e capacidade de oferta de serviços.

É inegável os grandes avanços que a APS, representada principalmente pela Estratégia Saúde da Família, apresentou nos últimos 25 anos. Houve expressiva redução da mortalidade infantil, redução de mortalidade evitável, redução de internações por condições sensíveis, entre outros avanços ${ }^{11-13}$. Por outro lado, percebe-se uma diminuição expressiva na velocidade de ganhos em saúde frente ao investimento público. Há diminuição da cobertura vacinal, perda de velocidade na redução da mortalidade infantil, uma grande proporção de internações hospitalares evitáveis, além de enorme dificuldade no manejo das doenças crônicas, no envelhecimento e, também no enfrentamento da sífilis e do HIV. Além do desafio em aumentar a efetividade da APS no SUS, sua eficiência também preocupa. Em estudo do Banco Mundial, foi observado que o nível de eficiência da atenção primária à saúde (APS) estava em torno de $60 \%$, com um desperdício anual de aproximadamente $\mathrm{R} \$ 9.3$ bilhões, somando os recursos dos três níveis de gestão ${ }^{14}$. 
Em paralelo a avaliações de indicadores de saúde, inúmeras investigações mediram a força dos atributos da APS brasileira nos últimos anos $^{15-19}$. É evidente que há muito a avançar para que a APS obtenha maior presença e extensão dos indicadores essenciais - acesso de primeiro contato, longitudinalidade, integralidade, coordenação - e derivados - orientação comunitária e familiar e competência cultural. A busca pelo fortalecimento dos atributos deve guiar a Política Nacional da APS e as decisões políticas a respeito, inclusive com mudanças no financiamento federal. Ademais, é necessário que, além da garantia de financiamento compatível com as atribuições e potencial da APS, os mecanismos de alocação de recursos federais para a APS e respectivos repasses aos entes Municipais sejam cuidadosamente estruturados a fim de que se constituam também como meio indutor do alcance de melhores resultados em saúde. Para tanto, é imprescindível que se institua um modelo misto de financiamento que leve em consideração um método de capitação ponderada por critérios de equidade, de pagamento por desempenho das equipes de Saúde de Família e de incentivos a ações estratégicas e prioritárias, semelhante a fee-for-service, tendo como exemplo, mas não como receita, os melhores sistemas de saúde baseados em APS do mundo ${ }^{20}$. Diante de tal contexto, esse artigo apresenta o novo modelo de financiamento para APS no Brasil, aprovado em Portaria Tripartite da Comissão Intergestores em 31 de outubro de 2019 e promulgada na Portaria Ministerial no 2.979 de 12 de novembro de $2019^{21}$.

\section{Método}

Para a construção de um novo modelo de financiamento da APS brasileira foi montado um time de mais de 60 pessoas vinculadas à Secretaria de Atenção Primária à Saúde do Ministério das Saúde, contando com apoio técnico de profissionais do Banco Mundial, da Universidade de Harvard, da Universidade Federal do Rio de Janeiro e da Universidade Federal do Rio Grande do Sul. Num primeiro momento foram revistos criticamente todos os tipos de repasses federais relacionados à APS. Em paralelo, revisão da literatura nacional e internacional apontou o melhor desenho de um modelo misto de financiamento da APS. A partir da definição de que o novo financiamento seria baseado em capitação ponderada com viés de equidade, de pagamento por desempenho e incentivos em ações estratégicas e prioritárias, iniciou-se a realização de inúmeras simulações que garantisse o maior ganho financeiro possível aos municípios brasileiros. Para tanto, aumento de R 2 bilhões no orçamento federal da APS em 2020 em comparação a 2019 foi garantido pelo Ministro da Saúde, Luis Henrique Mandetta, representando $11 \%$ de aumento real no orçamento, em pleno cenário de crise econômica.

\section{Novo Financiamento: composição}

As simulações de impacto do Novo Financiamento Federal para a Atenção Primária à Saúde (APS) computaram o efeito de: Capitação ponderada; Pagamento por desempenho (P4P); Incentivo para Ações Estratégicas; e Provimento de profissionais de saúde.

O cálculo das transferências para os municípios foi feito de acordo com a seguinte fórmula:

$\operatorname{Transf}_{i, t}=\operatorname{Cap}_{i, t}+\operatorname{Des}_{i, t}+\sum_{j=1}^{15} \operatorname{Inc}_{i, t, j}+\sum_{k=1}^{2} \operatorname{Prov}_{i, t, k}$

Onde: $\operatorname{Transf}_{\mathrm{i}, \mathrm{t}}=$ total da transferência federal para o município no período; Cap $_{\mathrm{i}, \mathrm{t}}=$ transferência por capitação para o município no período; Des $_{\mathrm{i}, \mathrm{t}}=$ total da transferência baseada em desempenho para o município no período $t ;$ Inc $_{i, t, j}$ $=$ incentivo aos programas/estratégias $\mathrm{j}$ para $\mathrm{o}$ município no período; $\operatorname{Prov}_{\mathrm{i}, \mathrm{t}, \mathrm{k}}=$ provimento de Agentes Comunitários de Saúde (ACS) do programa k para o município no período.

As simulações foram calculadas a partir das informações relativas aos municípios beneficiados pelos programas da APS, população cadastrada e suas características (beneficiário de programas sociais e idade). Os valores projetados para o orçamento para cada componente estão na Tabela 1.

Para fins de análise, os valores das transferências para os municípios no novo modelo de financiamento foram comparados com os valores de 2019 para cada município, a fim de estimar o impacto (perdas ou ganhos) do Novo Financiamento nas transferências federais para APS.

Capitação ponderada - comparada à soma dos recursos financeiros a seguir:

- PAB Fixo referente ao ano de 2019;

- 12 parcelas dos recursos financeiros referentes à competência financeira 08/2019, correspondentes a Equipes de Saúde da Família (eSF) e Núcleo Ampliado de Saúde da Família e Atenção Básica (NASF-AB), sem considerar suspensões por qualquer motivo (para os municípios que não informaram nenhuma eSF ou NASF na competência financeira 08/2019, foi considerado 
Tabela 1. Orçamento da APS (Sumário), 2019/20.

\begin{tabular}{lrr}
\hline \multicolumn{1}{c}{ Novos critérios de alocação } & $\mathbf{2 0 1 9}(\mathbf{4 3 . 0 0 0}$ eSF $)$ & $\mathbf{2 0 2 0}(\mathbf{4 6 . 6 0 0}$ eSF $)$ \\
\hline Capitação ponderada & $\mathrm{R} \$ 10.077 .779 .352$ & $\mathrm{R} \$ 10.684 .562 .796$ \\
Incentivo para Ações Estratégicas & $\mathrm{R} \$ 2.217 .854 .876$ & $\mathrm{R} \$ 3.011 .339 .009$ \\
Desempenho & $\mathrm{R} \$ 1.970 .672 .081$ & $\mathrm{R} \$ 1.865 .888 .397$ \\
Provimento (Agente Comunitário da Saúde) & $\mathrm{R} \$ 4.121 .410 .000$ & $\mathrm{R} \$ 4.845 .859 .200$ \\
Total & $\mathrm{R} \$ 18.387 .716 .309$ & $\mathrm{R} \$ 20.407 .649 .402$ \\
\hline
\end{tabular}

o valor referente à competência com maior número de equipes no ano de 2019);

- 6 parcelas do recurso financeiro referente à competência financeira 08/2019, correspondentes aos Gerentes.

Pagamento por desempenho - comparado a 12 parcelas dos recursos financeiros que os municípios fazem jus de acordo com a segunda lista de certificação do $3^{\circ}$ ciclo do PMAQ, sem considerar suspensões de qualquer tipo.

Incentivo para Ações Estratégicas:

- 12 parcelas dos recursos financeiros referentes à competência financeira 08/2019, correspondentes a Equipes de Saúde Bucal (ESB), Laboratório Regional de Prótese Dentária (LRPD), Unidade Odontológica Móvel (UOM), Centro de Especialidade Odontológica (CEO), Equipe de Saúde da Família Ribeirinha (ESFR), Unidade Básica de Saúde Fluvial (UBSF), Consultório na Rua, Academia de saúde, Microscopistas, Equipes de Atenção Básica Prisional e Saúde do Adolescente, sem considerar suspensões por qualquer motivo (para os municípios que não receberiam nada na competência financeira $08 / 2019$, foi considerada como referência a competência com maior valor no ano de 2019);

- Parcela única de 2019 do Programa Saúde na Escola (PSE);

- 6 parcelas do recurso financeiro referentes à competência financeira 08/2019, correspondentes ao Programa Saúde na Hora considerado apenas o custeio da Unidade de Saúde da Família aderidas e o impacto orçamentário no custeio das eSF e eSB quando estas participam do programa ( $\mathrm{R} \$ 3.565,00$ e $\mathrm{R} \$ 2.240,00$ respectivamente);

- Destaca-se que para as equipes de Saúde da Família Ribeirinhas foram considerados neste componente somente os valores referentes aos profissionais extras, unidades de apoio e embarcações, já que os valores referentes às equipes foram incluídos para comparação com a capitação ponderada.
Provimento de profissionais de saúde: para a simulação foram considerados apenas os valores referentes aos ACS para os quais foram calculadas 13 parcelas do recurso financeiro referentes à competência financeira 08/2019 sem considerar suspensões por qualquer motivo (para os municípios que não informaram nenhuma ACS na competência financeira $08 / 2019$, foi considerado o valor referente à competência com maior número de agentes no ano de 2019).

\section{Simulações do Novo Modelo de Financiamento da Atenção Primária}

\section{Capitação Ponderada}

\section{Parâmetro de cadastro}

A transferência de recursos financeiros do componente de capitação ponderada considera o quantitativo de pessoas cadastradas em equipes de Saúde da Família (eSF) ou equipes de Atenção Primária (eAP), ponderada por critérios de equidade. $\mathrm{O}$ parâmetro de pessoas cadastradas por equipe varia de acordo com o tipo de equipe e a tipologia do município considerando a classificação e caracterização dos espaços rurais e urbanos proposta pelo IBGE ${ }^{22}$ (Quadro 1).

Ponderação do cadastro (cálculo dos pesos)

Para a ponderação da capitação ponderada foram levados em conta três critérios de equidade: vulnerabilidade socioeconômica, ajuste demográfico e ajuste de distância. Os critérios de vulnerabilidade socioeconômica e ajuste demográfico atribuíram um peso diferenciado para pessoas cadastradas beneficiárias de programas sociais e dentro das faixas de idade que são consideradas com maior necessidade dos serviços de saúde. Esses critérios são definidos da seguinte forma:

- Vulnerabilidade Socioeconômica: população no município que recebe benefício do bolsa fa- 
Quadro 1. Parâmetro de cadastro por equipe - de acordo com o tipo de município.

\begin{tabular}{|c|c|c|c|}
\hline Tipologia rural-urbana IBGE & $\begin{array}{c}\text { Parâmetro de } \\
\text { cadastro para ESF }\end{array}$ & $\begin{array}{c}\text { Parâmetro de cadastro } \\
\text { para EAP - 20h }\end{array}$ & $\begin{array}{c}\text { Parâmetro de cadastro } \\
\text { para EAP - 30h }\end{array}$ \\
\hline 1 - Urbano & 4.000 pessoas & 2.000 pessoas & 3.000 pessoas \\
\hline 2 - Intermediário Adjacente & \multirow{2}{*}{2.750 pessoas } & \multirow{2}{*}{1.375 pessoas } & \multirow{2}{*}{2.063 pessoas } \\
\hline 3 - Rural Adjacente & & & \\
\hline 4 - Intermediário Remoto & \multirow{2}{*}{2.000 pessoas } & \multirow{2}{*}{1.000 pessoas } & \multirow{2}{*}{1.500 pessoas } \\
\hline 5 - Rural Remoto & & & \\
\hline
\end{tabular}

mília (BF) ou benefício de prestação continuada (BPC) ou benefícios previdenciários do INSS de até dois salários mínimos (INSS);

- Ajuste demográfico: população no município com até 5 anos e a partir de 65 anos de idade;

- Ajuste de distância: o ajuste de distância considera que os custos da provisão de APS nos municípios variam de acordo com sua distância de centros urbanos. Para este ajuste foi levado em conta a classificação e caracterização dos espaços rurais e urbanos de acordo com a metodologia proposta pelo IBGE para os municípios ${ }^{22}$.

Para os critérios de vulnerabilidade socioeconômica e ajuste demográfico foi atribuído um peso de 1,3 por pessoa. Isso significa que para cada pessoa considerada em vulnerabilidade socioeconômica ou dentro dessas faixas de idade o município será calculado $30 \%$ a mais do valor da capitação. Caso uma pessoa se enquadre tanto na vulnerabilidade socioeconômica quanto na faixa etária, o acréscimo de 30\% é aplicado apenas uma vez. O cálculo do peso levou em conta a razão de chances de uma pessoa em vulnerabilidade econômica ser dependente do Sistema Único de Saúde (SUS), ou seja, não ter plano de saúde.

Os pesos para cada município de acordo com a tipologia do IBGE consideraram a razão entre o parâmetro de cadastro por equipe dos municípios urbanos em relação às demais tipologias. Os valores dos pesos, por pessoa cadastrada, indicam a ponderação da pessoa no município. Sendo assim, em um município rural remoto ou intermediário remoto, a pessoa cadastrada possui peso 2, e receberá duas vezes mais por pessoa cadastrada do que um município urbano. Nos municípios intermediários adjacentes e rural adjacente, a pessoa cadastrada tem peso 1,45 vezes maior que a pessoa cadastrada no município urbano (Quadro 2).

\section{Cálculo dos pontos}

Para cálculo dos pontos de cada município foi estipulada primeiramente a meta de cadastro
Quadro 2. Peso por pessoa cadastrada - de acordo aos critérios de vulnerabilidade socioeconômica, ajuste demográfico e ajuste de distância.

\begin{tabular}{|l|l|}
\hline \multicolumn{1}{|c|}{ Critérios } & \multicolumn{1}{|c|}{$\begin{array}{c}\text { Peso por pessoa } \\
\text { cadastrada }\end{array}$} \\
\hline $\begin{array}{l}\text { Vulnerabilidade } \\
\text { socioeconômica ou } \\
\text { Ajuste demográfico }\end{array}$ & $\begin{array}{l}\text { Não se enquadra nos } \\
\text { critérios: } 1 \\
\text { Se enquadra nos critérios: } \\
1,3\end{array}$ \\
\hline Ajuste da distância & Urbano: 1 \\
& Intermediário adjacente: \\
& 1,45 \\
& Rural adjacente: 1,45 \\
& Intermediário remoto: 2 \\
& Rural remoto: 2 \\
\hline
\end{tabular}

municipal, que considera o número de equipes (eSF e eAP) implantadas multiplicado pelo parâmetro de cadastro por equipe de acordo com o tipo de equipe e a tipologia rural-urbana do município, sendo a meta de cadastro municipal limitada à população IBGE 2019.

Para o cálculo do número de equipes de cada município foram consideradas as eSF informadas pelo município na competência CNES 07/2019, limitadas ao quantitativo de eSF credenciadas pelo Ministério da Saúde, acrescidas das eAP potenciais de cada município considerando os profissionais cadastrados em estabelecimentos de saúde da Atenção Primária e informados no Sistema Nacional de Cadastro de Estabelecimentos de Saúde (SCNES) na competência CNES 07/2019. Para fins de cálculo do total de equipes de cada eAP foi considerada como $1 / 2 \mathrm{eSF}$.

Para estimar as potenciais equipes de eAP de cada município foram consideradas as duplas de médicos em enfermeiros com carga horária semanal de pelo menos 20 ou 30 horas num mesmo estabelecimento de Atenção Primária cadastrados no SCNES com os seguintes Códigos Brasileiros de Ocupação (CBO): Médico: 2251- 
42 ou 2251-70 ou 2251-30; Enfermeiro: 2235-65 ou 2235-05.

Foram utilizados ainda 3 critérios idênticos aos aplicados no método de cálculo de cobertura da Atenção Básica:

- Crítica da carga horária: não é contabilizada a carga horária de profissionais que tiverem registradas no SCNES quantidade de horas semanais superiores a 44 horas de outras horas, ou 60 horas ambulatoriais, ou 96 horas hospitalares, ou 120 horas resultantes da soma dessas categorias de horas semanais de trabalho;

- Lotação: profissionais não vinculados a equipes e lotados nos seguintes tipos de estabelecimentos no SCNES - 01 posto de saúde; 02 centros de saúde/unidades básicas de saúde; 32 unidades móveis fluviais; 40 unidades móveis terrestres;

- Natureza jurídica: 1000 - administração pública; 1015 - órgão público do poder executivo federal; 1023 - órgão público do poder executivo estadual ou do distrito federal; 1031 - órgão público do poder executivo municipal; 1040 - órgão público do poder legislativo federal; 1058 - órgão público do poder legislativo estadual ou do distrito federal; 1066 - órgão público do poder legislativo municipal; 1074 - órgão público do poder judiciário federal; 1082 - órgão público do poder judiciário estadual; 1104 - autarquia federal; 1112 - autarquia estadual ou do distrito federal; 1120 - autarquia municipal; 1139 - fundação federal; 1147 - fundação estadual ou do distrito federal; 1155 - fundação municipal; 1163 - órgão público autônomo federal; 1171 - órgão público autônomo estadual ou do distrito federal; 1180 - órgão público autônomo municipal; 1198 - comissão polinacional; 1201 - fundo público; 1210 - associação pública; 1228 - consórcio público de direito privado; 1236 - estado ou distrito federal; 1244 - município; 1252 - fundação pública de direito privado federal; 1260 - fundação pública de direito privado estadual ou do distrito federal; e 1279 - fundação pública de direito privado municipal.

Todas as duplas de profissionais identificadas foram classificadas com eAP modalidade I (com carga horária de 20 horas semanais), e contabilizadas como $1 / 2 \mathrm{eSF}$ para que sua distribuição entre os municípios fosse limitada ao teto de eSF já considerando o parâmetro de pessoas por equipe proposto pelo Novo Modelo de Financiamento. Ao total foram identificadas 2.809 eAP em 445 municípios.

A meta de cadastro municipal foi ponderada a partir dos pesos definidos acima, calculando o número de pontos por município. $\mathrm{O}$ ajuste con- siderou o número de pessoas em vulnerabilidade socioeconômica e nas faixas de idade prioritárias (peso da vulnerabilidade econômica ou ajuste demográfico $=1,3$ ) e a categoria do município de acordo com a classificação do IBGE (ajuste de distância).

Para definição das pessoas em vulnerabilidade socioeconômica e nas faixas de idade prioritárias foram realizados os seguintes passos:

- A população em vulnerabilidade socioeconômica foi estimada a partir do número de pessoas beneficiárias do bolsa família, do benefício de prestação continuada, e com aposentadoria do INSS menor do que dois salários mínimos no município, retirada uma sobreposição estimada de $3 \%$ entre estes beneficiários, identificando a proporção de pessoas em vulnerabilidade socioeconômica do município dentre a população total IBGE 2019. Foram utilizadas as seguintes fontes;

- Para o ajuste demográfico, a população nas faixas de idade prioritárias (menor ou igual a 5 anos e maior ou igual a 65 anos) de cada município foi obtida a partir da população IBGE 2018 aplicadas as proporções de cada faixa etária do IBGE 2010 e Sistema Nacional de Nascidos Vivos disponibilizada pela Secretaria Nacional de Vigilância em Saúde (SVS/MS);

- Entre a população em vulnerabilidade socioeconômica e a população nas faixas de idade prioritárias foi retirada a sobreposição utilizando o percentual de sobreposição entre as mesmas categorias identificadas para cada município no cadastro do Sistema de Informação em Saúde da Atenção Básica (SISAB) 23 ;

- A proporção final de pessoas que atentem aos critérios de vulnerabilidade socioeconômica ou ajuste demográfico dentre o total da população municipal foi aplicada à meta de cadastro municipal para posterior aplicação dos pesos destes mesmos critérios e do peso do ajuste de distância.

O total de pontos por município corresponde aos pontos referentes às pessoas cadastradas que atendem aos critérios de vulnerabilidade socioeconômica ou ajuste demográfico somados aos pontos referentes às pessoas cadastradas que não atendem aos critérios de vulnerabilidade socioeconômica ou ajuste demográfico, sendo esta somatória multiplicada pelo peso referente ao ajuste de distância. O total de pontos Brasil corresponde a somatória dos pontos de todos os municípios brasileiros conforme abaixo:

$$
\Sigma\left(\text { Ptos }_{i, t}\right)=\left[\Sigma\left(\text { Ptos }_{p v}\right)+\Sigma\left(\text { Ptos }_{p n v}\right)\right] \star \pi_{i}
$$


Onde: PTOS $_{i, t}=$ total dos pontos do município i no período $t$ considerando $100 \%$ da meta de cadastro municipal; PTOS $_{p, v}=$ pontos por pessoa cadastrada que estão nos critérios de vulnerabilidade econômica ou nas faixas de idade prioritárias (1.3 vezes o número de pessoas cadastradas dentro dos critérios); PTOS $_{p, n, v}=$ pontos por pessoa cadastrada fora dos critérios de vulnerabilidade econômica e nas faixas de idade prioritárias; $\pi_{\mathrm{i}}=$ pesos distância de acordo com a tipologia do IBGE (Quadro 1).

\section{Cálculo do valor de cada ponto}

Para cálculo do valor de cada ponto o orçamento previsto para 2020 para a capitação ponderada, excluído o orçamento reservado para o pagamento per capita fixo de transição para ano de 2020, foi dividido pelo total de pontos de todos os municípios brasileiros, conforme fórmula abaixo:

$$
\theta=\left[\frac{\$ \text { Capitação }_{2020}}{\sum\left(\text { Ptos }_{i, t}\right)}\right]
$$

Onde: $\theta=$ valor em reais de cada ponto; $\$ C a$ pitação ${ }_{2020}=$ orçamento estimado da capitação em 2020 (R 9.434 bilhões); PTOS $_{i, t}=$ total dos pontos do município i no período $t$ considerando $100 \%$ da meta de cadastro municipal.

O valor do ponto, ou seja, o valor per capita base nesta simulação corresponde à $\mathrm{R} \$ 51,35$ por ano.

\section{Cálculo do valor das transferências da capitação para cada município}

Para o cálculo da capitação ponderada de cada município foram consideradas apenas as eSF implantadas no município (eSF informadas no SCNES pelo município na competência CNES 07/2019, limitadas ao quantitativo de eSF credenciadas pelo Ministério da Saúde). As possíveis eAP identificadas no SCNES não foram consideradas visto que são apenas equipes estimadas e não implantadas na referida competência.

A partir das eSF implatadas foi calculada a meta real de cadastro municipal, multiplicando estas equipes ao parâmetro de cadastro por eSF por tipologia municipal. A seguir foi novamente calculado a proporção de pessoas que atendem aos critérios de vulnerabilidade socioeconômica ou ajuste demográfico dentro da meta real de cadastro municipal e aplicados os pesos referentes a estes critérios e ao ajuste de distância, estabelecendo o total de pontos por município.
O valor da capitação por município foi calculado a partir do número total de pontos do município multiplicado pelo valor de cada ponto $(\theta)$, conforme abaixo:

$$
\operatorname{Cap}_{i, t}=\theta^{*}\left[\Sigma\left(\text { Ptos }_{i, t}\right)\right]
$$

Onde: $\theta=$ valor em reais de cada ponto; $\operatorname{PTOS}_{i, t}=$ total dos pontos do município i no período $t$ considerando $100 \%$ da meta de cadastro municipal.

\section{Cálculo dos valores per capita fixo com base populacional - período de transição}

Para a transição do modelo atual para o novo modelo de financiamento foi estipulado que durante os 12 meses de 2020 parte do orçamento da capitação ponderada seria destinado a um pagamento fixo per capita com base populacional, o que corresponde a $\mathrm{R} \$ 1,250$ bilhões.

Para cálculo deste valor per capita fixo de base populacional o orçamento previsto de $\mathrm{R} \$$ 1,250 bilhões foi dividido entre a população do Brasil de acordo com o IBGE 2019, obtendo um valor de R \$ 5,95 por pessoa ano, e em seguida multiplicado pela população IBGE 2019 de cada município.

\section{Cálculos dos Incentivos para Ações Estratégicas}

O orçamento previsto para os Incentivos para Ações Estratégicas em 2020 é de R \$ 3,011 bilhões. A lista completa desses programas: Saúde na Hora, Equipe de Saúde Bucal (eSB), Unidade Odontológica Móvel (UOM), Centro de Especialidades Odontológicas (CEO), Laboratório Regional de Prótese Dentária (LRPD), Consultório na Rua (eCR), Unidade Básica de Saúde Fluvial (UBSF), Equipe de Saúde da Família Ribeirinha (eSFR), Microscopista, Equipe de Atenção Básica Prisional (EABP), Equipes de Saúde da Família (eSF) e Equipe de Atenção Primária (eAP) que atendem adolescentes em conflito com a Lei, Programa Saúde na Escola (PSE), Polo de Academia da Saúde, Informatização de equipe, Custeio aos municípios com Residência Médica e Multiprofissional.

O cálculo do valor das transferências federais aos municípios para cada programa está explicado abaixo.

Programa Saúde na Hora: os cálculos são baseados na lista de Unidades de Saúde da Família (USF) cuja adesão foi homologada em portaria (da $1^{\text {a a }} 8^{\mathrm{a}}$ portarias de adesão). O valor de transferência considerou 12 parcelas dos valores de cus- 
teio mensal das USF, e do impacto orçamentário da adesão das eSF e eSB participantes (para as eSF o valor utilizado foi $\mathrm{R} \$ 3.565,00$ mensais, e para as eSB o valor utilizado foi R\$ 2.240,00 mensais);

Informatização: os cálculos consideraram as eSF com prontuário eletrônico já implantado. O cálculo das transferências para esse programa utilizou a lista de eSF atualmente informatizadas (24.581 equipes) e o valor do incentivo para cada eSF de acordo com a tipologia IBGE: Rural Remoto R\$2.300,00; Intermediário Remoto e Rural Adjacente R\$ 2.000,00; Intermediário Adjacente e Urbano R\$ 1.700,00;

Incentivo de custeio aos municípios com Residência Médica e Multiprofissional: os cálculos utilizaram a estimativa de residentes por município que poderiam compor as eSF, considerando os programas de Residência Médica em Medicina de Família e Comunidade ou Residência Multiprofissional ou Uniprofissional em Odontologia e/ou Enfermagem com ênfase de Saúde da Família já financiada pelo Ministério da Saúde, e aplicando os valores de custeio mensal de R\$ $4.500,00$ por residente médico e $\mathrm{R} \$ 1.500,00$ por residente enfermeiro ou odontólogo.

Demais Programas: para esses, como não houve mudança nos critérios de repasse, o valor de 2019 foi replicado para 2020.

\section{Cálculo para Pagamento por Desempenho}

O orçamento previsto para 2020 do componente de pagamento por desempenho é de R\$ 1,865 bilhões. Entretanto parte deste orçamento será destinada a um período de transição entre modelos de financiamento e parte será destinada a implementação efetiva do novo componente de pagamento por desempenho.

Valores do período de transição: O período de transição para pagamento por desempenho será feito durante 8 meses, nos quais os municípios irão receber o equivalente ao valor de certificação do Programa Nacional de Melhoria do Acesso e da Qualidade (PMAQ) para os municípios participantes do referido programa no $3^{\circ}$ ciclo. Este período corresponde a um orçamento de R\$ 1,365 bilhões.

Valores pós período de transição: Após o período de transição, o pagamento por desempenho do Novo Financiamento se inicia nas últimas 4 competências do ano de 2020 correspondente a um orçamento de R $\$ 500$ milhões.

Foi realizado o cálculo do valor do desempenho por equipe. Para isso foram consideradas as eSF informadas por cada município na compe- tência CNES 07/2019, limitadas ao quantitativo de eSF credenciadas pelo Ministério da Saúde, acrescidas das eAP potenciais de cada município considerando os profissionais cadastrados em estabelecimentos de saúde da Atenção Primária e informados no Sistema Nacional de Cadastro de Estabelecimentos de saúde (SCNES) na competência CNES 07/2019. Para fins de cálculo do total de equipes cada eAP foi considerada como $1 / 2$ eSF. Em seguida o orçamento previsto foi dividido dentre todas as equipes a serem avaliadas pelo programa, conforme abaixo:

$$
\tau=\left[\frac{\text { \$ Desempenho }}{\mathrm{n} E \mathrm{Q} \text { D20(s) }}\right]
$$

Onde: $\$$ Desempenho $=$ orçamento previsto para as últimas 4 competências do ano de 2020 para o componente de desempenho (500 milhões); $\tau=$ valor do desempenho máximo por equipe; $n E Q=$ número total de equipes.

Para o cálculo do pagamento por desempenho pós transição de cada município foram consideradas apenas as eSF implantadas no município (eSF informadas no SCNES pelo município na competência CNES 07/2019, limitadas ao quantitativo de eSF credenciadas pelo Ministério da Saúde). As possíveis eAP identificadas no SCNES não foram consideradas visto que são apensas equipes estimadas e não implantadas na referida competência. Assim o valor do desempenho máximo por equipe foi multiplicado pelas eSF implantadas em cada município.

Cálculo do valor total das transferências do desempenho para cada município: Para cálculo do valor total das transferências do desempenho para cada município foram somados os calculados para o período de transição e para o período pós transição.

\section{Cálculo de Provimento de Profissionais de Saúde}

O orçamento previsto para o componente provimento de profissionais de saúde em 2020 é R\$ 4,845 bilhões, considerando apenas os Agentes Comunitários de Saúde. Para fins da simulação não foram considerados os recursos federais correspondentes aos programas de provimento médico, visto que estes não se tratam de repasse direto aos Fundos Municipais de Saúde.

Os valores referente aos ACS calculados para cada município em 2020 considerou a manutenção dos valores de 2019 ajustados com a correção do piso destes profissionais previstos para 2020 (de $\mathrm{R} \$ 1.250,00$ para $\mathrm{R} \$ 1.400,00$ mensais). 


\section{Comparação entre valores previstos para 2019 e 2020}

Após os cálculos dos valores previstos para cada município em 2019 e 2020 estes foram comparados para avaliação de possíveis perdas e ganhos. Os municípios que tiveram uma variação de 3\% a -3\% entre o ano de 2020 e 2019 foram considerados como manutenção dos recursos e classificados como faixa 0 , os municípios que ganharam entre $3 \%$ e $20 \%$ foram classificados como faixa 1, os municípios que ganharam mais do que $20 \%$ foram classificados como faixa 2, já os municípios que perderam de $-3 \%$ a $-20 \%$ foram classificados como faixa 3 , e aqueles com perda de $-20 \%$ ou mais foram classificados como faixa 4.

\section{Resultados}

A simulação dos resultados (Tabela 2) demonstrou que 1.354 de um total de 5.570 municípios

Tabela 2. Simulação do impacto do novo financiamento da APS nos municípios brasileiros para o ano de 2020 .

\begin{tabular}{llc}
\hline \multirow{4}{*}{ Total } & \multicolumn{1}{c}{ Variáveis } & Simulação \\
\cline { 2 - 3 } & Municípios Perdem & 1.354 \\
& Valor da Perda & 4.216 \\
& Valor do Ganho & $\mathrm{R} \$ 293.552 .435,29$ \\
\hline
\end{tabular}

(24\%) poderão apresentar perda de receitas em 2020. Para equilibrar esse cenário o Ministério da Saúde resolveu garantir às 12 competências de 2020 o valor do melhor PAB (fixo + Variável) do ano de 2019. O período de transição definido foi de um ano e, para tanto, será considerado o ano de 2020. Por outro lado, há ganho potencial de $\mathrm{R} \$ 2,3$ bilhões para mais de 4.200 municípios.

A fim de garantir esse ganho potencial, municípios devem ampliar o número de pessoas cadastradas nas equipes de Saúde da Família. Análise inicial dos cadastros válidos apontou para 93 milhões de pessoas cadastradas frente a um número calculado de cobertura que deveria ser de 148 milhões de pessoas. Os dados do Gráfico 1 demonstram que 26 das 27 unidades da federação apresentam um número de cadastrados menor que a cobertura estimada, possibilitando observar o tamanho do esforço de cadastramento que cada Unidade da Federação e seus municípios deverão fazer a fim de alcançar o ganho potencial de recursos financeiros para o ano de 2020 .

\section{Discussão}

O novo modelo de financiamento da APS implementado pelo Ministério da Saúde está proposto em três dimensões: capitação ponderada com viés de equidade individual e contextual, incentivo a ações e programas estratégicos e pagamento por desempenho. Essa mudança rompe com o modelo anterior baseado na população municipal -

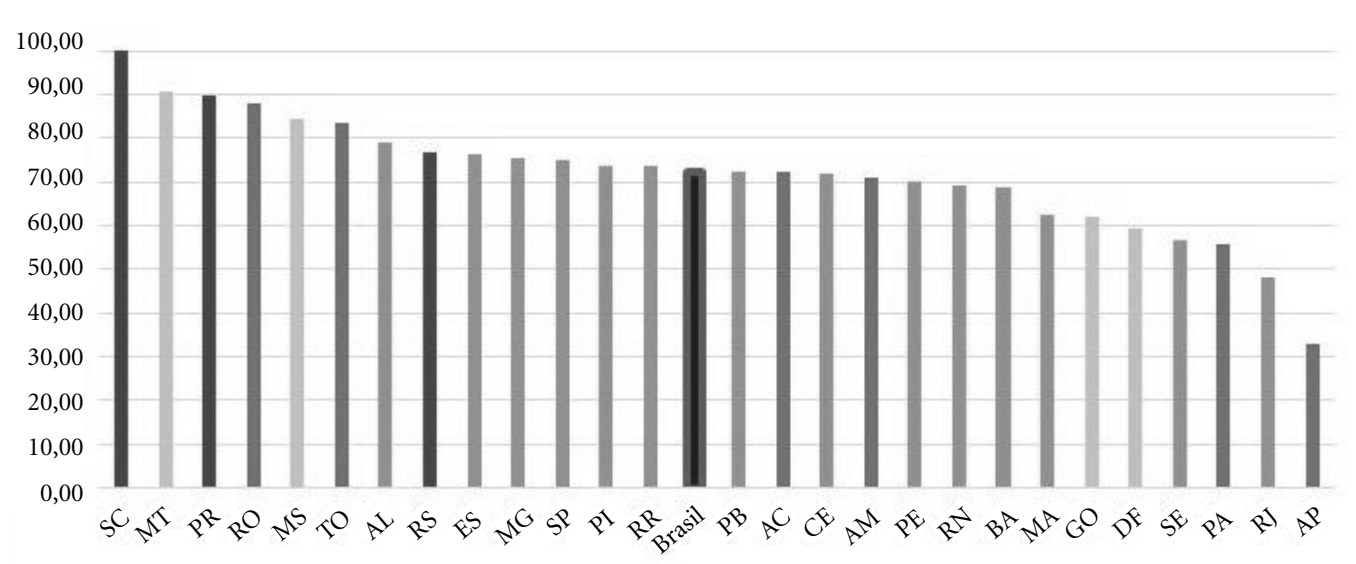

Gráfico 1. Proporção de cobertura Populacional (\%) de ESF (3.450 por equipe) pela Quantidade de pessoas Cadastradas (SISAB) por UF e Brasil. 
PAB Fixo (único repasse não condicionado, sem atualização frequente). Além disso, diminui os componentes de pagamento por incentivo a programas específicos repassados a partir da existência dos serviços - verificada através dos sistemas de informação. Essa reforma objetiva induzir as equipes a trabalhar com maior responsabilização pela população adscrita a partir do cadastramento das pessoas às equipes, aumentando a responsabilização das mesmas com seus pacientes e com melhoria da qualidade do cuidado.

Atualmente, existem 90 milhões de pessoas cadastradas em equipes de saúde da família. A cobertura potencial é de 148.674.300 milhões de pessoas cobertas ${ }^{24}$. Esses dados demonstram a dificuldade de acesso a serviços de saúde da população excluída dos cuidados primários de saúde. A ponderação do cadastramento aproxima mais a APS do princípio da equidade a medida que pondera positivamente os mais vulneráveis socialmente, idosos e crianças até cinco anos. Mais grave que o baixo número de pessoas cadastradas é o fato de cerca de 30 milhões de pessoas que vivem com apoio do Bolsa Família, do Benefício de prestação Continuada e de benefícios previdenciários de até 2 salários mínimos encontrarem-se entre os não-cadastrados. Uma verdadeira afronta ao princípio da universalidade e da equidade presentes na Constituição Federal na Lei 8.0801.

Para Smith e Rice ${ }^{25}$ há duas razões principais para se apostar num modelo de capitação, relacionadas à equidade e eficiência. Os argumentos de equidade tendem a refletir um requisito para garantir acesso igual aos cuidados de saúde (para necessidades de saúde iguais), priorizando acesso aos que possuem maiores necessidades em saúde. Os objetivos de eficiência estão implícitos na maioria dos esquemas de capitação, no sentido de que são incorporados a um sistema orçamentário que busca tornar prestadores mais responsivos às questões dos custos e benefícios de suas ações. Os autores apontam ainda a existência de diversos critérios que historicamente vêm sendo utilizados para ponderar a capitação: demográficos, étnicos, incapacitação para o trabalho, localização geográfica, morbidade e mortalidade, além de outros fatores sociais ${ }^{24}$. Modelos de financiamento por capitação são encontrados em diversos países como Reino Unido, Austrália, Canadá, Espanha, Suécia, Noruega, Holanda, Nova Zelândia, entre outros.

O Reino Unido financia seus serviços de saúde por capitação ponderada desde os anos 70, quando utilizou a forma Crossman's com base em idade, sexo, utilização de serviços e necessidades em saúde que passou a ser aprimorada pelo Resource Allocation Working Party (RAWP), tornando ainda mais explícito o princípio de equidade no modelo, sendo estendida para a APS nos anos $80^{20}$. Nos últimos 40 anos, a fórmula de capitação ponderada utilizada no Reino Unido foi alterada de acordo com a melhoria da qualidade dos dados registrados, maior possiblidade de granularidade das avaliações e uso de milhares de dados dos pacientes também foram importantes para melhoria contínua da forma de calcular a capitação ponderada, mantendo o foco na equidade do acesso.

Modelos de pagamento por desempenho (P4P) na APS são adotados em países como Austrália, Portugal, Turquia, Reino Unido, Nova Zelândia, entre outros. A Austrália adotou o P4P nos anos 90, como alternativa a um modelo de APS baseada em pagamento por serviços/programas considerado indutor de fragmentação. $\mathrm{O}$ novo programa é baseado em indicadores voltados a saúde materno-infantil, manejo de condições crônicas de saúde, qualidade da prescrição, uso de $e$-health, reforço das práticas de enfermagem, acesso aos serviços em horários diferenciados, saúde do idoso, saúde rural entre outros ${ }^{25}$. A Nova Zelândia adotou o pagamento por desempenho para conseguir resolver uma agenda de condições de saúde eleita como prioridade de saúde no ano de 2000 que envolvia redução de obesidade, de tabagismo, de depressão, de risco cardiovascular e de diabetes. Para tanto, elegeu 21 indicadores para seu programa obtendo melhora em todos eles ${ }^{26}$.

No Reino Unido, o Quality Outcome Framework (QOF) para pagamento por desempenho foi implementado objetivando aumentar a produtividade, redesenhar serviços para os pacientes, melhorar os serviços prestados na APS, criar a cultura e governança para melhoria da APS. Em 2012, o QOF apresentava 142 indicadores divididos em 4 grandes áreas: clínica (maior parte dos indicadores e voltados para qualidade da clínica da APS), organizacional (informações e registro dos pacientes, treinamento clínico do profissional), experiência do paciente com o serviço e serviços adicionais a APS. Desde o início do QOF em 2004, foi possível observar uma melhoria dos indicadores inicial com uma estabilização em níveis satisfatórios dentro do parâmetro estabeleci$\mathrm{do}^{27-34}$. Embora a experiência internacional seja heterogênea quanto ao pagamento por desempenho, é decisiva quando observamos a melhora dos indicadores em especial de imunização, saúde materno-infantil e doenças crônicas ${ }^{28-30}$. 
O Brasil apostará em 2020 em 7 indicadores para pagamento por desempenho, com incremento gradual de indicadores até alcançar $21 \mathrm{em}$ 2021. A totalidade dos 21 indicadores abrangerá ações relacionadas à saúde materno-infantil, às condições crônicas, às infecções sexualmente transmissíveis, à saúde mental, às internações sensíveis à APS, à fidelização das pessoas às Unidades de Saúde por meio do Net Promoter Score, à qualidade da relação médico-paciente por meio do PDRQ9 e da força dos atributos da APS por meio do PCATool-Brasil.

A semelhança entre os modelos citados e a reforma do financiamento da APS brasileira em curso não é por acaso. Para desenhar a reforma atual, modelos internacionais foram profundamente estudados e adaptados à realidade brasileira. Busca pela equidade, técnica, base científica e sensibilidade política foram as marcas do desenvolvimento do novo modelo de financiamento. Para a definição do modelo final houve amplo debate com a sociedade, tanto no Congresso Nacional, como em 23 Unidades da Federação com participação democrática de mais 10.000 gestores e integrantes das equipes de gestão das secretarias municipais de saúde. O CONASEMS, por meio da participação dos COSEMS, de sua equipe técnica e diretoria tem ampla participação na definição do modelo final, com considerações de maior relevância e que certamente tornaram o modelo final muito mais adequado à realidade do SUS. Além disso, essa movimentação representa importante estratégia de participação, credibilidade e aumento da capacidade de governabilidade em estratégias fundamentais para a consolidação de políticas públicas na APS ${ }^{27}$.

O Brasil optou pelo modelo misto de financiamento da APS. Assim como Reino Unido, o modelo envolve capitação ponderada, pagamento por desempenho e pagamento para oferta de serviços específicos. No modelo saxão a capitação ponderada representa $52 \%$ do volume do financiamento e o pagamento por desempenho 14\%; no modelo brasileiro a capitação ponderada é de $52 \%$ e o pagamento por desempenho $9 \%$ para o orçamento de $2020^{31}$. Essa opção ocorre por se reconhecer no Brasil a necessidade de se induzir a existência de alguns serviços específicos como o Saúde na Hora que mantém as UBS trabalhando em horário ampliado, as equipes de saúde bucal, InformatizaAPS que institui o financiamento federal como contrapartida ao envio de informações das equipes de Saúde da Família por meio de prontuários eletrônicos, incentivo para residência em Medicina de Família e Comuni- dade e a residência Multiprofissional ${ }^{35-37}$. Esses incentivos representam $15 \%$ do financiamento. O restante são recursos destinados a provimento de profissionais e ações de promoção de saúde e de atenção a populações especificamente vulneráveis, como as populações ribeirinhas.

Para Starfield ${ }^{38}$, os sistemas de saúde possuem como metas principais otimizar a saúde da população por meio do emprego do mais avançado conhecimento sobre a causa das enfermidades, manejo das doenças e maximização da saúde e minimizar as disparidades entre subgrupos populacionais de modo que determinados grupos não estejam em desvantagem sistemática em relação ao seu acesso aos serviços de saúde e ao alcance de um ótimo nível de saúde. A APS é o modo mais eficiente para atingir tais metas, por ser a entrada preferencial nos sistemas de saúde e ser baseada em atributos claramente definidos como acesso, longitudinalidade, integralidade, coordenação do cuidado, orientação familiar, orientação comunitária e competência cultu$\mathrm{ral}^{38}$. Esta, quando organizada sob a luz de seus atributos, proporciona melhora de acesso aos serviços necessários; qualidade do atendimento; um maior enfoque na prevenção, redução de cuidados especializados desnecessários e potencialmente prejudiciais ${ }^{39}$.

O novo modelo de financiamento proposto pela Portaria Ministerial no 2.979 de 12 de novembro de $2019^{21}$, em conjunto com as demais ações desenvolvidas pela Secretaria de Atenção Primária à Saúde (Saúde na Hora, InformatizaAPS, Médicos pelo Brasil, Incentivo a Residência Medicina de Família e Comunidade e Residência Multiprofissional), busca a inclusão de 50 milhões de pessoas que não estão cadastradas junto as ESF, implementa a capitação ponderada corrigindo, dessa forma, distorções de acesso aos mais vulneráveis, possibilita uma reorganização do cuidado pois induz uma identificação mais adequada das pessoas vinculadas a cada equipe de saúde da família, impõe a melhoria de indicadores buscando melhores resultados no cuidado, motiva o uso de prontuários eletrônicos do paciente qualificando as informações do paciente e permitindo um cuidado longitudinal e coordenado mais adequado, todas essas ações com objetivo final de fortalecer os atributos da APS.

\section{Considerações finais}

Ao trazer o financiamento da APS brasileira ao século XXI, o Ministério da Saúde e a equipe da 
Secretaria de Atenção Primária à Saúde pretendem fortalecer os atributos da Atenção primária com o objetivo de tornar concreto os princípios da Universalidade e da Equidade do SUS. Ao ofertar maiores repasses financeiros federais àqueles municípios e equipes que mais e melhor trabalham em prol da saúde das pessoas reforçase a reforma do Estado brasileiro em direção a uma prestação de serviços públicos que verdadeiramente atenda aos anseios da população.

\section{Colaboradores}

E Harzheim, OP D’Avila, LG Ramos e CMJ Santos participaram da concepção da proposta, análise dos dados, discussão dos dados observados e revisão do artigo. D Carvalho, LE Silva, LGM Costa, CRH Cunha e L Pedebos participaram do desenvolvimento do método, análise dos dados e resultados.

\section{Agradecimentos}

Agradecemos a Dirceu Ditamar Klitzke e Olívia Lucena Medeiros, servidores da Secretaria de Atenção Primária à Saúde, pela contribuição no desenvolvimento e aperfeiçoamento da proposta. 


\section{Referências}

1. Brasil. Lei no 8.080, de 19 de setembro de 1990. Dispõe sobre as condições para a promoção, proteção e recuperação da saúde, a organização e o funcionamento dos serviços correspondentes e dá outras providências. Diário Oficial da União 1990; 20 set.

2. Brasil. Lei no 8.142, de 28 de dezembro de 1990. Dispõe sobre a participação da comunidade na gestão do Sistema Único de Saúde (SUS) e sobre as transferências intergovernamentais de recursos financeiros na área da saúde e dá outras providências. Diário Oficial da União 1990; $31 \mathrm{dez}$.

3. Brasil. Portaria n ${ }^{\circ} 545$, de 20 de maio de 1993. Estabelece normas e procedimentos reguladores do processo de descentralização da gestão das ações e serviços de saúde, através da Norma Operacional Básica - SUS 01/93. Diário Oficial da União 1993; 20 maio.

4. Brasil. Portaria no 2.203, de 5 de novembro de 1996. Aprova a NOB 1/96, a qual redefine o modelo de gestão do Sistema Único de Saúde. Diário Oficial da União 1996; 5 nov.

5. Brasil. Portaria GM/MS no 1.882 , de 18 de dezembro de 1997. Estabelece o Piso da Atenção Básica - PAB e sua composição. Diário Oficial da União 1997; 18 dez.

6. Brasil. Portaria no 3.925, de 13 de novembro de 1998 . Aprova o Manual para Organização da Atenção Básica no Sistema Único de Saúde. Diário Oficial da União 1998; 17 nov (republicado).

7. Brasil. Portaria no 95 , de 26 de janeiro de 2001. Aprova a Norma Operacional da Assistência à Saúde - NOASSUS 01/2001. Diário Oficial da União 2001; 26 jan.

8. Brasil. Portaria no 204, de 29 de janeiro de 2007. Regulamenta o financiamento e a transferência dos recursos federais para as ações e os serviços de saúde, na forma de blocos de financiamento, com o respectivo monitoramento e controle. Diário Oficial da União 2007; 29 jan.

9. Brasil. Portaria no 3.992, de 28 de dezembro de 2017. Altera a Portaria de Consolidação no 6/GM/MS, de 28 de setembro de 2017, para dispor sobre o financiamento e a transferência dos recursos federais para as ações e os serviços públicos de saúde do Sistema Único de Saúde. Diário Oficial da União 2017; 28 dez.

10. Brasil. Lei complementar $n^{\circ} 141$, de 13 de janeiro de 2012. Regulamenta o $\$ 3^{\circ}$ do art. 198 da Constituição Federal para dispor sobre os valores mínimos a serem aplicados anualmente pela União, Estados, Distrito Federal e Municípios em ações e serviços públicos de saúde; estabelece os critérios de rateio dos recursos de transferências para a saúde e as normas de fiscalização, avaliação e controle das despesas com saúde nas 3 (três) esferas de governo; revoga dispositivos das Leis nos 8.080, de 19 de setembro de 1990, e 8.689, de 27 de julho de 1993; e dá outras providências. Diário Oficial da União 2012; 16 jan.

11. Kringos DS, Boerma W, van der Zee J, Groenewegen P. Europe's Strong Primary Care Systems Are Linked To Better Population Health But Also To Higher Health Spending. Health Affairs 2013; 32(4):686-694.

12. Rocha R, Soares R. Evaluating the impact of community-based health interventions: evidence from Brazil's Family Health Program. Health Econnomics 2010; 129(51):126-158.
13. Bastos ML, Menzies D, Hone T, Dehghani K, Trajman A. Correction: The impact of the Brazilian family health on selected primary care sensitive conditions: A systematic review. PLoS One 2017; 12(12):e0189557.

14. Banco Mundial. Um ajuste justo: Análise da eficiência e equidade do gasto público no Brasil [página na Internet]. 2017 [acessado 2019 Nov 28]. Disponível em: https://www.worldbankorg/pt/country/brazil/publication/brazil-expenditure-review-report

15. Mesquita Filho M, Luz BSR, Araújo CS. A Atenção Primária à Saúde e seus atributos: a situação das crianças menores de dois anos segundo suas cuidadoras. Cien Saude Colet 2014; 19(7):2033-2046.

16. Carvalho VCHS, Rossato SL, Fuchs FD, Harzheim E, Fuchs SC. Assessment of primary health care received by the elderly and health related quality of life: a crosssectional study. BMC Public Health 2013; 13:605.

17. Ibañez N, Rocha JSY, Castro PC, Ribeiro MCSA, Forster AC, Novaes MHD, Viana ALA. Avaliação do desempenho da atenção básica no Estado de São Paulo. Cien Saude Colet 2006; 11(3):683-703.

18. Harzheim E, Hauser L, Pinto LF. Avaliação do grau de orientação para Atenção Primária em Saúde: a experiência dos usuários das Clínicas da Família e Centros Municipais de Saúde na cidade do Rio de Janeiro (relatório final da pesquisa PCATool - Rio-2014). Porto Alegre: UFRGS; 2015.

19. Pinto LF, Harzheim E, Hauser L, D’Avila OP, Gonçalves MR, Travassos P, Pessanha R. A qualidade da Atenção Primária à Saúde na Rocinha - Rio de Janeiro, Brasil, na perspectiva dos cuidadores de crianças e dos usuários adultos. Cien Saude Colet 2017; 22(3):771781.

20. Buck D, Dixon A. Improving the allocation of health resources in England - How to decide who gets what. Ideas that change Health Care. Londres: The Kings Fund; 2013.

21. Brasil. Portaria no 2.979, de 12 de novembro de 2019. Institui o Programa Previne Brasil, que estabelece novo modelo de financiamento de custeio da Atenção Primária à Saúde no âmbito do Sistema Único de Saúde, por meio da alteração da Portaria de Consolidação $n^{\circ}$ 6/GM/MS, de 28 de setembro de 2017. Diário Oficial da União 2019; 13 nov.

22. Instituto Brasileiro de Geografia e Estatística (IBGE). Classificação e caracterização dos espaços rurais e urbanos do Brasil: uma primeira aproximação. Rio de Janeiro: IBGE; 2017.

23. Brasil. Sistema de Informação em Saúde para Atenção Básica (SISAB) [página na Internet]. [acessado 2019 dez 01]. Disponível em: https://sisab.saude.gov.br/

24. Brasil. Ministério da Saúde (MS). Secretaria de Atenção à Saúde. Departamento de Atenção Básica. Qualificação dos Indicadores do Manual Instrutivo para as equipes de Atenção Básica (Saúde da Família, Saúde Bucal e Equipes Parametrizadas) e NASF. Brasília: MS; 2015.

25. Rice P, Smith P. Approaches to Capitation and Risk Adjustment in Health Care: An International Survey. York: Centre for Health Economics, The University of York; 1999. 
26. Chasing C, Chi Y-L, Smith P, Borowitz M, Thomson S. Paying for Performance in Health Care - Implications for health system performance and accountability. Genebra: European Observatory on Health Systems and Policies; 2014.

27. Saltman R, Rico A, Boerma W. Primary Care in the Driver's Seat? Organizations Reform in European Primary Care. Genebra: European Observatory on Health Systems and Policies; 2006.

28. Martin J, Associates Limited. Evaluation of the PHO performance programme: final report. Auckland: Martin J and Associates Limited; 2008.

29. Ministry of Health New Zealand. New Zealand health strategy. Wellington: Ministry of Health; 2000.

30. Campbell SM, Roland MO, Middleton E, Reeves D. Improvements in the quality of clinical care in English general practice: longitudinal observational study. BMJ 2005; 331(7525):1121.

31. Campbell S, Reeves D, Kontopantelis E, Middleton E, Sibbald B, Roland M. Quality of primary care in England with the introduction of pay for performance. N Engl J Med 2007; 357(2):181-190.

32. Campbell S, MacDonald R, Lester H. The experience of pay for performance in English family practice: a qualitative study. Ann Fam Med 2008; 6(3):228-234.

33. Doran T, Fullwood C, Gravelle H, Reeves D, Kontopantelis E, Hiroeh U, Roland M. Pay-for-performance programs in family practices in the United Kingdom. N Engl J Med 2006; 355(4):375-384.

34. Pike C. An Empirical Analysis of the Effects of GP Competition. Co-operation and Competition Panel Working Paper Series; 2010.

35. Brasil. Portaria no 930, de 15 de maio de 2019. Institui o Programa "Saúde na Hora", que dispõe sobre o horário estendido de funcionamento das Unidades de Saúde da Família, altera a Portaria no 2.436/GM/ MS, de 2017, a Portaria de Consolidação no 2/GM/ MS, de 2017, a Portaria de Consolidação no 6/GM/ MS, de 2017, e dá outras providências. Diário Oficial da União 2019; 15 maio.
36. Brasil. Portaria no 2.983 de 11 de Novembro de 2019. Institui o Programa de Apoio à Informatização e Qualificação dos Dados da Atenção Primária à Saúde - Informatiza APS, por meio da alteração das Portarias de Consolidação no 5/GM/MS e n 6/GM/MS, de 28 de setembro de 2017. Diário Oficial da União 2019; 11 nov.

37. Brasil. Portaria no 3.510 de 18 de dezembro de 2019. Altera a portaria de Consolidação no 6/GM/MS, de 28 de setembro de 2017, para instituir incentivo financeiro de custeio adicional mensal para municípios com equipes de saúde integradas a programas de formação profissional no âmbito da Atenção Primária à Saúde. Diário Oficial da União 2019; $18 \mathrm{dez}$.

38. Starfield B. Atenção primária: equilíbrio entre necessidades de saúde, serviços e tecnologia. Brasília: UNESCO, MS, 2002.

39. Starfield B, Shi L, Macinko J. Contribution of primary care to health systems and health. Milbank Q 2005; 83(3):457-502.

Artigo apresentado em 02/12/2019

Aprovado em 20/12/2019

Versão final apresentada em 22/12/2019 